\title{
Los valores del sector público y la supuesta supremacía administrativa del sector privado*
}

\begin{abstract}
Argyriades, Demetrios**
** Realizó estudios de economía, política y administración pública en París I - Sorbonne. Fue consultor de la OECD. Funcionario de la ONU, en ésta su carrera está dividida entre administración interna de la organización y cooperación técnica en administración pública. Profesor de Administración Pública en R. F. Wagner Graduate School de la Universidad de Nueva Cork. Autor de diversos trabajos en tres idiomas. E-mail: argyriades@un.org
\end{abstract}

\section{Resumen}

El trabajo tiene como propósito discutir la validez de los modelos de gerencia privada impuestos en la administración pública durante las dos últimas décadas del siglo XX, bajo la denominación de Nueva Gerencia Pública. Los resultados dan cuenta que la crisis desencadenada por el ataque del 11 de septiembre y la implosión de Enron Corporation y Arthur Andersen conducen al cuestionamiento del sector privado y llaman la atención sobre el sector público. En este sentido se discuten las diferencias entre la gerencia privada y la pública, el costo del traslado de los modelos gerenciales privados, la necesidad de profesionalismo y determinados valores en el servicio público y la falsa representación de los ciudadanos como clientes consumidores. Se concluye argumentando la necesidad de regulación y defensa del interés general.

Palabras clave: Administración pública, gerencia pública, gerencia privada, servicio público, valores.

\section{Public sector Values and the Supposed Administrative Supremacy of the Private Sector}

\begin{abstract}
The purpose of this paper is to discuss the validity of private sector management models in public administration imposed on public administration during the last decade's $f$ the XXth Century under what has been called the New Public Management model. The results of the analysis indicate that the crisis unleashed by the Septembe 11 incident in the United States and the implosion of the Enron Corporation and the Arthur Anderson Accounting Firm lead to a questioning of the private sector and greater attention on the
\end{abstract}


part of the public sector. In this sense the differences between private and public sector management are discussed, as well as the cost of transfering private management models, the need for professionalism and certain values in the public service sector, and the lack of citizen, client and consumer representation. The conclusion argues the need for regultation and the defense of public interest.

Key words: Public administration, public management, private management, public service, values.

Recibido: 03-09-29. Aceptado: 03-11-24

\section{I ntroducción}

En el año 2001, dos crisis que marcaron época pusieron en duda no sólo la validez de las doctrinas gerenciales prevalecientes, sino también sus valores. La primera crisis corresponde a los trágicos eventos ocurridos en Nueva York el 11 de septiembre; la segunda fue provocada por la implosión de Enron Corporation. Arthur Andersen, la gran compañía de auditores y consultores en administración, se encontró gravemente implicada en este tema, el cual se convirtió en un gran escándalo público. Ciertamente, la revelación de que World Com, Xerox, Sisco y muchas otras firmas se encuentran en situaciones similares parece sugerir que el problema corresponde al sistema, que no es algo del azar y que Enron/Arthur Andersen representan sólo la punta de un iceberg de corrupción.

Prima facie, estos dos eventos no tienen mucho en común. Ambos, de diferente forma, han servido para llamar la atención sobre la importancia, significado y función del Estado y del servicio público. Asimismo, han servido para plantear interrogantes sobre el sector privado, cuyas afirmaciones de superioridad han sido tomadas de manera axiomática. Hasta hace un año, el sector privado no sólo estaba en su mejor momento, sino que su ejemplo proyectaba al mundo un modelo a seguir para la reforma del sector público. El lenguaje utilizado para publicitar este caso no podría ser más categórico: “El modelo tradicional de administración es obsoleto y ha sido efectivamente reemplazado por un nuevo modelo de gerencia pública. Este cambio representa una transición, desde un modelo burocrático hacia un modelo de mercado gerencial muy relacionado al sector privado. Las reformas gerenciales representan una transformación, no sólo de la gerencia pública sino también de las relaciones entre el mercado y el gobierno; gobierno y burocracia y burocracia y ciudadanía" (Hughes, 1998).

En teoría y en la práctica, las implicaciones de esta postura fueron drásticas y extensas. 
La reforma de las rutas del gobierno era sólo el inicio. "Reducir sus fronteras" no necesariamente implicaba reducciones generalizadas de los gastos gubernamentales, como una forma de reordenar radicalmente programas y prioridades. Incluso en tiempos de paz, la defensa, la ley y el orden recibieron un fuerte incentivo. Sin embargo, los sectores de educación y bienestar social no tuvieron la misma suerte. El resultado comprometió tres décadas de progreso hacia el propósito de lograr un mundo más equitativo, divulgar oportunidades de manera más amplia y mejorar el concepto de derechos humanos. En los niveles nacional e internacional, los objetivos de justicia social fueron relegados. Con el incremento de la pobreza y el desempleo aumentaron también a paso acelerado las desigualdades en la riqueza, el poder y los conocimientos (UNDP, 1999; NU, 2000:8).

El "modelo de mercado", promovió de manera agresiva la contracción del Estado. Los encargados de las reformas halagaron las virtudes de la administración que proponía reducciones drásticas utilizando la privatización, liberación de normas, etc. bajo el supuesto no comprobado de que cualquier acción emprendida por el gobierno podría ser ejecutada de manera más eficiente por el sector privado, libre de obstáculos del Estado. Dichas medidas se siguieron o defendieron incluso en países donde el marco institucional apropiado era virtualmente inexistente; en donde las alternativas del sector privado para servicios proporcionados por el Estado no podían ponerse a disposición del público a un precio asequible y en donde la cultura gerencial predicada sobre trabajo ético y respeto por el imperio de una ley estaba aún por desarrollarse.

Los resultados disfuncionales de este enfoque no tardaron en salir a la superficie. Sin embargo, hasta hace poco, la ortodoxia que prevalecía podía compararse con las heridas causadas durante un proceso de "transición", o como el precio a pagar por el fracaso al aceptar los desafíos de "cambio" y "globalización". Particularmente a comienzos de los años noventa, se dio mucho significado específico a esos términos combinados. Ellos fueron integrados como si lo que denotaran fueran verdades evidentes por sí mismas. Los términos "cambio", “modernización", “transición” y “globalización", representados en términos lineales, hacían referencia a tendencias, las cuales eran consideradas como casi universales e impulsadas por fuerzas históricas que apuntaban irreversiblemente hacia una sola dirección: el triunfo de los mercados, la contracción del Estado, la desaparición gradual de las culturas tradicionales débiles y la convergencia de valores. El decaimiento del Estado, profetizado por Vladimir Lenin, se convirtió en el punto central de las reformas neo-liberales y en los años 90 obtuvo aceptación como la expresión del Estado postbienestar, un nuevo orden mundial después de la Guerra fría. 


\section{2. ¿Se diluyen las diferencias entre Gerencia Pública y Privada?}

Una derivación de esta creencia fue la llamada "Nueva Gerencia Pública” (NGP), la cual en realidad no era tan nueva. Apoyándose en la Teoría de la Opción Pública, descartó todos los conceptos tales como interés público o general, el cual, desde el Siglo de las Luces, proporcionó las bases del Estado y del servicio público. Del mismo modo, la administración pública fue puesta en cuestión. El lema frecuentemente citado "gerencia es gerencia", sugería de una manera muy explícita que las estrictas distinciones entre los roles y las funciones de los administradores del sector público y del sector privado eran cosas realmente del pasado. En línea con este enfoque, enfatizado en best-sellers tales como "Reinventando el Gobierno" por D. Osborne y T. Gaebler (1994), se encontraba el mensaje de que la "administración regulada" necesitaba ser reemplazada por la gerencia empresarial; "los resultados sobre proceso" exigidos y las prácticas contrarias a la búsqueda de la eficiencia debían ser condenadas o minimizadas.

“Dejar que los gerentes administren" se convirtió en el lema del movimiento. La eficiencia y la eficacia, junto con la economía, recibieron el más alto lugar dentro de la jerarquía de los valores. Por el contrario, el imperio de la ley y los procesos justos , perdieron territorio. A pesar de contar con mucho apoyo, especialmente desde hace algunos años, aquellos que apoyan la NGP los consideran como elementos de "las estructuras tradicionales de la gobernabilidad que necesitan ser minimizados ya que (...) pueden interferir con la efectividad y la eficacia de la administración pública en términos económicos" (Sommermann (2001: 2).

El énfasis en las tres "E" (economía, eficiencia y eficacia) fue inequívoco. Opacó la función de la ley, de las ciencias políticas, la psicología, la filosofía y de otros contribuyentes a la teoría y práctica de la administración pública. A la luz de este enfoque, la dicotomía entre la administración del sector privado y la administración pública fue considerada como "obsoleta".

Si "gerencia es gerencia", en otras palabras, la misma para todos los campos de su actividad ya sea pública o privada, ¿podríamos entonces concluir que la distinción histórica entre estas dos áreas de la gerencia es algo del pasado y que el servicio público es una "profesión que está muriendo?". De ser así, ¿debe- ríamos reformar nuestras políticas y prácticas para la contratación en el sector público, el campo profesional y la capacitación de modo que se aproximen más a los modelos del sector privado que nos inspiraron? ¿Deberíamos revisar los valores que deseamos inculcar; revisar nuevamente las normas y patrones que en nuestra opinión los funcionarios deberían tomar como ejemplo? 
En verdad no estoy de acuerdo. Sin embargo, esto no significa que un acercamiento de patrones, formas y prácticas juiciosamente seguidas no pueda rendir un beneficio mutuo, sino que la convergencia en algunas áreas puede ser igualada para otros, con diferencias, las cuales deben ser reconocidas y entendidas. Dichas diferencias, refinadas con el correr del tiempo y en diferentes contextos, han marcado la identidad separada de la profesión en el servicio público. Ellas apuntan sus reclamos hacia un cierto nivel de autonomía y llevaron la dirección de la reforma del servicio público a una cierta distancia desde el campo político y los objetivos con fines de lucro.

Al revisar nuevamente los valores de la profesión en el servicio público, necesitamos que se nos recuerden las condiciones que rodean sus génesis y evolución. La conversión de una pequeña banda de cortesanos y criados en una profesión totalmente desarrollada al servicio del bienestar público, puede ser considerada como uno de los mayores logros institucionales de los últimos doscientos años. No se logró de la noche a la mañana, pero las fuerzas que encabezaron la transformación incluyeron presiones por la democracia, los intereses por el bienestar público y la necesidad de promover la integridad en la vida pública. La lucha contra la corrupción, el tráfico de influencias y la arbitrariedad tiene un impacto definitivo en la formación de las instituciones de la profesión del servicio público. Específicamente, justifica la introducción de políticas y prácticas por las cuales aún se le conoce:

- Exámenes competitivos abiertos a fin de asegurar no sólo la alta calidad de los resultados sino también la índole de la profesión como dominio público.

- Reconocimiento del mérito como la mayor consideración en la promoción, contratación y remuneración del personal; la primacía del conocimiento, el desempeño y la experiencia sobre las relaciones personales (amiguismo) o los favores.

- Una medida de autonomía en el ejercicio de funciones y juicios independientes junto con responsabilidad sobre las decisiones y acciones.

- Un alto comportamiento ético en la conducción del sector público, respaldado inter alia por procesos transparentes y por el imperio de la ley.

\section{El costo de la privatización de la administración pública}

A la luz del desarrollo y del progreso logrado contra todos los pronósticos durante los últimos doscientos años, la campaña contra el Estado y la profesión en el sector público, conducida principalmente durante los años 80 y 90, representó una regresión. Debe 
enfatizarse que lo que se ha buscado en nombre de las tres "E" y la "guerra contra la burocracia" ha significado:

- Un retorno a prácticas de tráfico de influencias.

- Entrometimiento en el espacio público y privatización del dominio público.

- Prominencia de particularismo sobre un criterio universal en la conducción de asuntos públicos.

- Incremento substancial en la corrupción y baja credibilidad en las instituciones públicas como guardianes del bienestar público.

No deberíamos sorprendernos de que tal exacerbación de comportamiento anti-social, ilustrado recientemente por la ráfaga de mega escándalos (Enron, World Com, Arthur Andersen), ha sido frecuentemente acompañada por una retórica proveniente de aquellas fuentes que persistentemente han defendido la "gerencia empresarial" sobre la “administración reglamentada". Los propósitos públicos de "outsourcing” y "dejar que los gerentes administren", evidentemente tienen un precio muy alto. El costo de la Nueva Gerencia Pública, aparte de incrementar la pobreza y la desigualdad en diversas partes del mundo, ya sea países desarrollados o en vías de desarrollo, ha creado mayormente un interés decreciente en la vida pública, un número muy bajo de votantes, problemas agudos en la contratación de personal en el sector público, así como corrupción endémica. La respuesta de la NGP a dichos desafíos emergentes ha sido casi la misma: mayor asimilación de objetivos y valores y "estrategias anticorrupción".

\section{Profesionalismo y valores del sector público}

Los intentos para castigar a los malhechores son oportunos y altamente necesarios. Sin embargo, las medidas punitivas por si solas, incluso si se comprueba que son efectivas, no hacen frente al tema de la ética en el servicio público, un punto que toca el centro de lo que hace el servicio, a quién sirve y por qué se le llama profesión. Se ha discutido en muchas oportunidades que "no existe una sola profesión al seno del gobierno", sino muchas. Los gobiernos emplean todo tipo de personas, tales como médicos, meteorólogos, maestros, abogados, personal de seguridad, así como aquellos cuyo campo de trabajo no encaja bajo un solo nombre". Cada grupo obedece a preceptos particulares, los cuales se han adaptado y aun continúan gobernando la práctica de su trabajo. Lo que podrían tener en común es la búsqueda de la excelencia y una obligación compartida para extender las fronteras del conocimiento en sus respectivos campos. En tiempos de cambios rápidos y de un verdadero progreso vertiginoso, esto es lo que hace de la capacitación una tarea de aprendizaje de por vida. 
La dedicación a los objetivos de estudio y auto-superación se encuentran al mismo nivel que el entredicho para utilizar la competencia y el conocimiento sólo para propósitos legítimos, esencia del profesionalismo en cualquier campo. Sin embargo, el sector público y el trabajo para el gobierno crean sus propios requerimientos. En palabras de Naomi Caiden (1999:80): “Los profesionales del gobierno tienen ciertos atributos en común. Se desenvuelven en un marco de trabajo institucional y legal, el cual les ofrece sus propias desventajas y oportunidades. Comparten el compromiso de utilizar sus habilidades para el beneficio del público, en contraposición a la ganancia privada, a cambio de una remuneración fija. Están sujetos a la responsabilidad pública por sus acciones".

El concepto de profesionalismo, aplicado al servicio gubernamental o al servicio público en general, tiene menos que ver con la esencia de las funciones desarrolladas que con el ambiente donde se desempeñan. Como se mencionó anteriormente, esto se refiere a las condiciones, las cuales son comunes a todos los funcionarios y al objetivo de sus servicios, en lugar de referirse a la naturaleza de las actividades que desarrollan. Las condiciones se encuentran en textos legales, constitucionales y reguladores, los cuales especifican la forma en la que debe ejercerse la autoridad investida en las instituciones del sector público. Sin embargo, el público objetivo de sus servicios, que normalmente está conformado por ciudadanos comunes y políticos, tiene expectativas adicionales. Dichas expectativas están llenas de experiencias y de tradiciones históricas y contribuyen a dar forma a la imagen del sector público y a todas sus relaciones.

Sería justo discutir las enormes diferencias existentes entre la naturaleza del ambiente en el que operan el sector privado y el gobierno, respectivamente. Dichas diferencias contradicen el argumento de la NGP de que "gerencia es gerencia" y, por lo tanto, que los deberes de los empresarios del sector privado y de los funcionarios del gobierno es realmente indistinguible. Aunque existan analogías, las desventajas y las oportunidades que enfrentan ambos grupos no son las mismas. Los empresarios operan en un ambiente de mercado, en el que la supervivencia económica y el éxito se predican con base en la eficiencia y en la eficacia. Se goza de obediencia a la ley, pero, como sugieren los últimos eventos, rara vez es escrupulosamente observada. Aunque las motivaciones varíen, el afán de lucro representa la fuerza motriz que lleva a la gente a la acción.

En el sector privado, las tres "E" (economía, eficiencia y eficacia) encabezan la escala de valores. Contrariamente, en el sector público éstos toman su lugar dentro de un amplio marco de lineamientos y normas. De todos ellos, el interés público y la propiedad democrática son los más importantes. En el pasado, la lealtad a la monarquía y a sus 
ministros se interpretaba como un principio absoluto. En la actualidad, se trata de una subordinación a los líderes políticos y obediencia a sus órdenes, pero siempre dentro del marco de la ley, los procesos justos, la propiedad constitucional y la moralidad.

La adhesión a los principios de gobernabilidad democrática también origina sus propias desventajas. Dichos "valores fundamentales" para las relaciones nacionales e internacionales, modificados por la Declaración del Milenio (Naciones Unidas, 2000a), incluyen: Libertad, Igualdad, Solidaridad, Tolerancia por la diversidad, Respeto por el medio ambiente y Responsabilidad compartida. Los objetivos son consistentes con estos valores. Los redactores de dicho documento identificaron los objetivos y les "otorgaron especial significación". Los más importantes son: paz y el imperio de la ley; desarrollo y erradicación de la pobreza, protección del medio ambiente; democracia; buena gobernabilidad y derecho humanos.

La importancia relativa que toman estos valores y objetivos en ambos sectores marca la diferencia entre ellos. En el sector privado, exhortan a los empresarios a adherirse a un comportamiento de moda, el cual es socialmente responsable. Ellos no pueden hacer más. Sin embargo, en el sector público, los valores son un componente esencial en cualquier código de ética y tienen una influencia formativa en las relaciones entre servidores públicos y ciudadanos. Esta diferencia fundamental hace inválido el reclamo de la NGP en el sentido "que los gobiernos no sólo deben adoptar las técnicas de la gerencia empresarial sino (...) también los valores de los negocios (León y Demhardt, 2002).

\section{La falsa representación de los ciudadanos como clientes consumidores}

En la Nueva Gerencia Pública, los ciudadanos son consumidores. Ciertamente, el ciudadano-consumidor, puede ser considerado como un elemento esencial del nuevo paradigma. La metáfora del mercado sobre la que se predica tiene valor ya que apela a la respuesta de los ciudadanos y contribuyentes. Sin embargo, la analogía se detiene y la metáfora puede ser engañosa. Los valores de democracia y aquellos de los mercados globales pueden converger de manera parcial, pero raramente coinciden. Sería problemático encontrar entre estos últimos un lugar para alguno de los principios enfatizados en la Declaración del Milenio.

Necesitamos recordar que la ciudadanía es un derecho adquirido desde el nacimiento. La participación igualitaria en los procesos democráticos está basada en derechos individuales, los cuales están estipulados en la constitución o en otros reglamentos. En teoría, no se debería permitir ninguna distinción a este respecto. Por el contrario, en el 
sector privado el dinero gobierna el acceso del cliente y su relación con el mercado. La medida de su valor depende de su poder adquisitivo. Mientras que compradores y vendedores pueden escoger uno o el otro, el acceso de un ciudadano a los servicios proporcionados por el gobierno está basado en derechos y necesidades. Un Estado democrático no se encuentra en libertad de favorecer o discriminar. Sería apropiado discutir que sin importar cuáles sean sus méritos, el paradigma del mercado tiene serias limitaciones cuando se refiere a gerencia pública en las sociedades democráticas.

Este paradigma no enfatiza dos de los principales valores de la gobernabilidad democrática: igualdad e inclusión. Su congruencia con el principio del imperio de la ley y de proceso justo también debe ser cuestionada. Lo que es más, la representación falsa de ciudadanos como consumidores va al mismo nivel que la tendencia de igualar la esencia del gobierno con la prestación de servicios. Este énfasis en el servicio no sólo reduce la función y el alcance del servicio público, sino que también amenaza con trivializar a los ciudadanos ubicándolos de manera colectiva al final del proceso político, comparándolo con los clientes.

Exhortar a los trabajadores públicos a “iproducir, producir, producir! sólo evoca la réplica “idame, dame, dame!" (Argyriades, 2002:57; Vigoda, 2002). En nivel nacional, subnacional e internacional necesitamos reafirmar los verdaderos valores de la ciudadanía que complementan aquellos del sector público. Ciertamente, la ciudadanía democrática equivale a mucho más que mero consumismo y servicio público, a más que una satisfacción de codicia. No es sólo la gran participación en los asuntos de la gobernabilidad lo que constituye la esencia de la ciudadanía democrática sino también es la promoción de estilos de vida viables con producción sustantiva y patrones de consumo lo que necesita ser considerado como la esencia del servicio público responsable.

\section{En defensa de la regulación y del interés general}

El espíritu cívico complementa y refuerza el servicio público pero no obvia su función o importancia. La auto-regulación ayuda, pero, como indican los escándalos que involucran a Arthur Andersen en complicidad con Enron, World Com, esto no es suficiente. No es substituto de la regulación. Dichos escándalos sirvieron para exponer la falacia de los extremos y la necesidad de perspectivas más balanceadas. Los méritos de la desregulación para la descentralización y desburocratización no están en cuestión. Por otro lado, lo que parece es que ellas no proporcionan beneficios sin la presencia de un "centro de apoyo" integrado por profesionales competentes y servidores públicos virtuosos 1 . 
Uno de los roles más importantes de un "centro de apoyo" que justifique completamente la necesidad de trabajadores públicos capacitados, profesionales y virtuosos es una tarea muy importante en la articulación, promoción, defensa y representación del interés público o general. El concepto de interés general fue fuertemente criticado en los años 80 y 90 por los adherentes de la Teoría de la Opción Pública, quienes, por lo tanto, también cuestionaron el valor del servicio público $\underline{2}$. Recientemente, ambos conceptos marcaron un retorno.

En un mundo de cambios vertiginosos y de las complejas sociedades prismáticas en las que vivimos, la tarea de reconciliar puntos de vista divergentes e intereses conflictivos no es la única dimensión en la tarea sin final de definir y redefinir lo que es de interés general. Cada vez más esta función se ve complicada por la necesidad de incorporar dos consideraciones: progreso a largo plazo vs. exigencias a corto plazo e intereses globales o internacionales vs. intereses nacionales o locales.

Durante las Reuniones de Expertos del Programa de Naciones Unidas en Administración Pública y Finanzas (Naciones Unidas, 1997; 2000; 2002) dichos puntos se discutieron ampliamente. Probablemente la XV Reunión de Expertos (Naciones Unidas, 2000) de manera más enfática solicitó “la proclamación del día del Servicio Público, el cual celebrará el valor y la virtud del servicio a la comunidad en los niveles nacional, subnacional y global". (Recomendación 3). Asimismo, recomendó que los trabajadores públicos aprendan a "trabajar en los niveles nacional, subnacional e internacional" (Recomendación 19).

Del mismo modo, la última Reunión de Expertos (julio de 2002) destacó la interdependencia de problemas, campos y disciplinas en los niveles nacionales, subnacionales e internacionales. Reconociendo que no existen proyectos universales para las reformas y destacando que el papel de la buena gobernabilidad: "debe tomarse en cuenta la cultura y las circunstancias de los Estados Miembros", la Reunión enfatizó:

- La importancia de asesoría en temas de políticas.

- La necesidad de un intercambio global de información "identificando buenas prácticas y haciendo hincapié en las lecciones aprendidas y en la reducción de riesgos".

- La necesidad de acelerar el único cambio observable de "una orientación externa a una interna hacia todos los segmentos de la sociedad". 
Para los trabajadores públicos, las recomendaciones, que tocaron temas de valores y capacitación, tuvieron sentido. Dichas recomendaciones hicieron hincapié en la importancia de una capacitación interdisciplinaria y de un conocimiento profundo más allá de sólo conocer la mera técnica. Asimismo, sugieren la necesidad de redefinir conceptos consagrados, tales como lealtad y obediencia. La cooperación internacional efectiva para un desarrollo global sustentable, con justicia, equidad y paz requiere de los trabajadores públicos no sólo un conocimiento profundo y el entendimiento de temas complejos, sino también de una mentalidad significativamente distinta a lo que se promovió en el pasado. En un mundo de gran complejidad y de creciente interdependencia, la integridad y el compromiso deben ir en línea con la tolerancia y la moderación; los deberes de obediencia y subordinación, con los principios de no hacer el mal, respetar los derechos humanos y "hablar con la verdad".

Los registros del anterior siglo y de los recientes meses, llenos de tristes ejemplos de horrores perpetrados por funcionarios "que siguen órdenes", deberían alertarnos de los peligros no sólo de una ciega sumisión, sino también de una lealtad sin límites ante cualquier causa 3 . "Mi país, correcto o no" como también "resultados sobre proceso", sugieren un tipo de actitud y una mente "de una sola vía", que son la antítesis del liderazgo en el servicio público, así como de la ética profesional. En lugar del común "Sí, señor Ministro", lo que las organizaciones públicas necesitan en los niveles nacional e internacional es una asesoría basada en principios; informada, equilibrada, consistente, responsable e imbuida del liderazgo que ofrecen los verdaderos profesionales.

\section{Notas}

* Conferencia Magistral presentada en el VIII Foro de Investigación. Congreso Internacional de Contaduría, Administración e Informática. Organizado por la UNAM (México) del 29 al 31 de octubre de 2003. Publicación autorizada por los organizadores del evento.

1. La necesidad de un centro de apoyo para una descentralización exitosa es común a todos los países. Basada en la constitución y en otros textos pertinentes, un marco legislativo claro y consistente debería establecer los fundamentos para la distribución de funciones y competencias entre el Estado y el autogobierno local y entre los diversos niveles de autogobierno local. Dicha distribución de funciones no impide una cooperación cercana entre ellos" (Naciones Unidas, 2000b).

2. "En el movimiento de reinvención del gobierno, el "servicio" es el enemigo a ser vencido por los ciudadanos que eligen y por trabajadores públicos que preparan las opciones públicas" (Fredericson, 1996: 265). 
3. Una idea similar fue expresada por el Profesor Yehezkel Dror (2001) a favor de moverse del tradicional concepto de "Razón de Estado" hacia un concepto más amplio de "razón de humanidad".

\section{Referencias Bibliográficas}

1. Argyriades, Demetrios (2002), “General Report" en International Institute of Administrative Sciences. Governance and Public Administration in the 21st Century: New Trends \& New Techniques. Proccedings. Atenas.

2. Caiden, Naomi (1999), “Public Service Professionalism in performance Measurement and Evaluation" en Public Service in Transition: Enhancing its Role, Professionalism, Ethical Values and Standards, Naciones Unidas, DESA/SEM/97/1. New York.

3. Dror, Yehezkel (2001), Capacity to Govern: a Report to the Club of Rome. Londres.

4. Frederickson, H. G. (1996), “Comparing the reinventing Government Movement with the New Public Administration". En: Public Administration Review, Vol. 56, No. 3. Washington.

5. Hughes, Owen E. (1998), Public Management and Administration: An I ntroducción, 2da. Edición, Nueva York: St. Martin's Press, p. 242.

6. León, Linda de y Dernhardt, Robert G. (2002), The political Theory of reinvention" Public Administration Review, Vol. 60 No.2, Washington.

7. Naciones Unidas (NU) (1997), Reporte de Expertos del Programa E/ 1997/ 86. New York.

8. Naciones Unidas (NU) (2000), Reporte de Expertos del Programa E/ 2000/ 66. New York.

9. Naciones Unidas (NU) (2000a), Declaración del Milenio. A/ 55/ L.2. New York.

10. Naciones Unidas (NU) (2000b), “Descentralization and Conditions for Sucess: Lessons from Central and Eastern Europe \& the C.I.S." ST/ESA/PAD/SER. E/7. New York. pp 144. 
11. Naciones Unidas (UN) (2002), Reporte de Expertos del Programa E/ 2002/ 26. New York.

12. Osborne, David y Gaebler, Ted (1994), La Reinvención del Gobierno. La influencia del espíritu empresarial en el sector público. Ediciones Paidós. Barcelona. España.

13. Sommermann, Karl Peter (2001), "The Rule of Law and Public Administration in a Global Setting". I nforme al Congreso I nternacional de Ciencias Administrativas, 913 julio. Atenas.

14. UNDP (1999), Human Devenlopment Report. Washington.

15. Vigoda, Eran (2002), “From responsiveness to Collaboration; Governance, Citizens and the Next Generation of Public Administration". En: Public Administration Review, Vol. 62, No.5, Washington. pp. 527-540. 